\title{
Investigation of Viscous Fluid in a Rotating Disk
}

\author{
R.Lakshmi ${ }^{1}$, M.Muthuselvi ${ }^{2}$ \\ ${ }^{I}$ Assistant Professor, Department of Mathematics, PSGR Krishnammal College for women, Coimbatore, \\ Tamil Nadu, India \\ ${ }^{2}$ Assistant Professor, Department of Mathematics, Dr.SNS Rajalakshmi College of Arts and Science, \\ Coimbatore, Tamil Nadu, India.
}

\begin{abstract}
In this paper, the unsteady viscous flow produced by a sudden coincidence of the axes of a disk and the fluid at infinity rotating with the uniform angular velocity $\Omega$ has been studied. The exact solution of the governing equation has been obtained using Laplace transform technique. The velocity field and the shear stress components that rare related to the force components in the $x$ and $y$-directions exerted by the fluid on the disk are discussed. The exact solutions are also verified by another solution obtained and that is valid at small times instants. The fluid motions are depicted through graphs using MATLAB.
\end{abstract}

Key Words: viscous fluid, rotating disk, angular velocity, Laplace transform

\section{Introduction}

The rotating disk is a popular geometry has studying different flows, because of its simplicity and the fact that it represents a classical fluid dynamics problem. It is a subject of wide spread practical interest in connection with stream turbines, gas turbines, pumps and other rotating fluid machines. This flow parading is also be used to investigate the momentum and heat and mass transfer characteristics of Newtonian and NonNewtonian fluids.

The flow due to an infinite rotating disk is one of the classical problems in fluid mechanics which was first introduced by Von karman. He show that the Navier Strokes equation a steady flow on the Newtonian incompressed fluid due to disk rotating far from other solid surfaces can be reduced the set of ordinary differential equations. These equations can be solved by an approximate integral method.

Erdogan (1997) was the first to study the unsteady floe generated by non-coaxial rotation suddenly while a disk and a fluid at infinity are initially rotating about a common axis. He obtained an exact solution to this type of flow for a Newtonian fluid. Pop (1979) was the first to consider the unsteady flow produced by a disk and a fluid at infinity. He considered the problem for a Newtonian fluid a studied the unsteady flow induced when the disk and the fluid at infinity start impulsively rotate with the same angular velocity about non coincident axes. Kasiviswanathan and Rao (1987) presented an exact solution of the unsteady Navier-Stokes equations for the floe produced by an eccentrically rotating porous disk oscillating in its own plane and the fluid at infinity. Erdogan (1997) studied the unsteady viscous flow resulting from rotation about non-coaxial axes while the disk and the fluid at infinity are initially rotating about a common axis. Hayat et al. (1999) obtained an exact analytic solution for the unsteady disk in its own plane. They also discussed the unsteady viscous flow induced by the oscillations of a porous disk in its own plane. They also discussed the unsteady viscous flow due to the porous disk oscillating and the fluid at infinity rotating about an axis parallel to their first rotation axis. Erdogan (2000) studied the flow due to non coaxial rotation of a disk oscillating in its own plane and a viscous fluid at infinity. Hayat et al. (2001) examined Erdogan's work (1997) for a porous disk in the presence of a magnetic field. Siddique ct al. (2001) studied the same problem for second grade fluid. However, they considered small values of the elastic parameter.

In this mainly we studied about the unsteady flow of a Newtonian fluid resulting from rotation about a common axis while a disk and the same angular velocity about non-coincident axes. The disk and the fluid at infinity rotate with the same angular velocity about non- coincident axes. The disk and the fluid at infinity rotate with the same angular velocity throughout the flow.

\section{Mathematical Formulation}

Let us consider a Newtonian fluid filling the semi infinite space $\mathrm{z} \geq 0$ in a Cartesian coordinate system. The axis of rotation of the disk located at $\mathrm{z}=0$ and that of the fluid at infinity are in the plane $\mathrm{x}=0$. The disk and the fluid at infinity are initially rotating with the same angular velocity $\Omega$ about the $\mathrm{z}$ - and $\mathrm{z}$ axes, respectively, and the distance between the axes is denoted by 1 . The disk suddenly starts to rotate with its initial angular velocity about the $\mathrm{z}$-axis. Therefore, the initial and boundary conditions are

$$
\begin{array}{ll}
\mathrm{u}=-\Omega \mathrm{y}+\hat{\mathrm{f}}(\mathrm{z}), \mathrm{v}=\Omega \mathrm{x}+\hat{\mathrm{g}}(\mathrm{z}), & \mathrm{w}=0 \text { at } \mathrm{t}=0 \text { for } \mathrm{z} \geq 0, \\
\mathrm{u}=-\Omega(\mathrm{y}-\ell), \mathrm{v}=\Omega \mathrm{x}, & \mathrm{w}=0 \text { at } \mathrm{z}=0 \text { for } \mathrm{t}>0,
\end{array}
$$




$$
\mathrm{u}=-\Omega(\mathrm{y}-\ell), \mathrm{v}=\Omega \mathrm{x}, \quad \mathrm{w}=0 \text { at } \mathrm{z}=\infty \text { for } \mathrm{t} \geq 0,
$$

Where $\mathrm{u}, \mathrm{v}, \mathrm{w}$ denote the velocity components along the $\mathrm{x}, \mathrm{y}, \mathrm{z}$-directions, respectively. The functions $\hat{\mathrm{f}}(\mathrm{z})$ and $\hat{g}(\mathrm{z})$, obtained by courier(1972), are given by

$$
\hat{\mathrm{f}}(\mathrm{z})+\mathrm{i} \hat{\mathrm{g}}(\mathrm{z})=\Omega \ell\left(1-\mathrm{e}^{-\mathrm{kz}}\right)
$$

Where $\mathrm{i}=\sqrt{-1}, \mathrm{k}=(1+\mathrm{i}) \sqrt{\frac{\Omega}{2 \mathrm{v}}}$, and $\mathrm{v}$ is the kinematic viscosity of the fluid. The solution shown by Eq. (2) is summarized in the Appendix.

Thus it seems reasonable to try a solution of the form

$$
\mathrm{u}=-\Omega \mathrm{y}+\mathrm{f}(\mathrm{z}, \mathrm{t}), \quad \mathrm{v}=\Omega \mathrm{x}+\mathrm{g}(\mathrm{z}, \mathrm{t}), \quad \mathrm{w}=0,
$$

This seems that the flow is a result of superposition, in each $z=$ constant plane, of a rigid body rotation with the angular velocity $\Omega$ about the z-axis and of a time dependent rigid body translation that changes from plane to plane with the velocity $\{\mathrm{f}(\mathrm{z}, \mathrm{t}) ; \mathrm{g}(\mathrm{z}, \mathrm{t}) ; 0\}$ in a Cartesian coordinate system. Using Eqs(1-3) and (5) we have

$$
\begin{gathered}
\mathrm{f}(\mathrm{z}, 0)=\hat{\mathrm{f}}(\mathrm{z}), \mathrm{g}(\mathrm{z}, 0)=\hat{\mathrm{g}}(\mathrm{z}) \quad \text { for } \mathrm{z} \geq 0, \\
\mathrm{f}(0, \mathrm{t})=\Omega \ell, \quad \mathrm{g}(\infty, \mathrm{t})=0 \quad \text { for } \mathrm{t}>0, \\
\mathrm{f}(\infty, \mathrm{t})=\Omega \ell, \mathrm{g}(\infty, \mathrm{t})=0 \quad \text { for } \mathrm{t} \geq 0,
\end{gathered}
$$

Substituting Eq. (5) into the Navier-stokes equations, one obtains

$$
\begin{aligned}
& \frac{1}{\rho} \frac{\partial \mathrm{p}}{\partial \mathrm{x}}=\Omega^{2} \mathrm{x}+\left(\mathrm{v} \frac{\partial^{2} \mathrm{f}}{\partial \mathrm{z}^{2}}-\frac{\partial \mathrm{f}}{\partial \mathrm{t}}+\Omega \mathrm{g}\right), \\
& \frac{1}{\rho} \frac{\partial \mathrm{p}}{\partial \mathrm{y}}=\Omega^{2} \mathrm{y}+\left(\mathrm{v} \frac{\partial^{2} \mathrm{~g}}{\partial \mathrm{z}^{2}}-\frac{\partial \mathrm{g}}{\partial \mathrm{t}}-\Omega \mathrm{f}\right), \\
& \frac{1}{\rho} \frac{\partial \mathrm{p}}{\partial \mathrm{z}}=0,
\end{aligned}
$$

Where $\rho$ is the density of the fluid and $p$ is the modified pressure. Equations (9-11) give

$$
\begin{aligned}
& \mathrm{v} \frac{\partial^{2} \mathrm{f}}{\partial \mathrm{z}^{2}}-\frac{\partial \mathrm{f}}{\partial \mathrm{t}}+\Omega \mathrm{g}=\mathrm{C}_{1}(\mathrm{t}), \\
& \mathrm{v} \frac{\partial^{2} \mathrm{~g}}{\partial \mathrm{z}^{2}}-\frac{\partial \mathrm{g}}{\partial \mathrm{t}}-\Omega=\mathrm{C}_{2}(\mathrm{t})
\end{aligned}
$$

Since the fluid at infinity has no shear stress, we find that $C_{1}(t)=0$ and $C_{2}(t)=-\Omega^{2} l$ with the help of Eq. (4c).Introducing $F(z, t)=f(z, t)+i g(z, t)$ and using Eqs. (12-13), we get

$$
v \frac{\partial^{2} \mathrm{~F}}{\partial \mathrm{z}^{2}}-\frac{\partial \mathrm{F}}{\partial \mathrm{t}}-\mathrm{i} \Omega \mathrm{F}=-\mathrm{i} \Omega^{2} \mathrm{l}
$$

If we introduce the following dimensionless variables

$$
\mathrm{F}=\frac{\mathrm{f}}{\Omega \mathrm{l}}+\mathrm{i} \frac{\mathrm{g}}{\Omega \mathrm{l}}-1, \zeta=\sqrt{\frac{\Omega}{2 \mathrm{v}}} \mathrm{z}, \tau=\Omega \mathrm{t}
$$

Then Eq. (14) and the conditions (6-8) become

And

$$
\frac{\partial^{2} \mathrm{~F}}{\partial \zeta^{2}}-2 \frac{\partial \mathrm{F}}{\partial \tau}-2 \mathrm{iF}=0,
$$

Solution of the problem

$$
\begin{aligned}
\mathrm{F}(0, \tau) & =0(\tau>0), \\
\mathrm{F}(\infty, \tau) & =0(\tau \geq 0), \\
\mathrm{F}(\zeta, 0) & =-\mathrm{e}^{-(1+\mathrm{i}) \zeta}(\zeta \geq 0) .
\end{aligned}
$$

Setting

We have

$$
F(\zeta, \tau)=H(\zeta, \tau) e^{-i \tau},
$$

$$
\frac{\partial^{2} \mathrm{H}}{\partial \zeta^{2}}-2 \frac{\partial \mathrm{H}}{\partial \tau}=0,
$$

With the conditions as follows:

$$
\begin{aligned}
H(0, \tau) & =0(\tau>0), \\
H(\infty, \tau) & =0(\tau \geq 0) \\
H(\zeta, 0) & =-e^{-(1+i) \zeta}(\zeta \geq 0) .
\end{aligned}
$$

Let the Laplace transform of $\mathrm{H}(\zeta, \tau)$ be $\overline{\mathrm{H}}(\zeta, \mathrm{s})$, so that 


$$
\overline{\mathrm{H}}(\zeta, \mathrm{s})=\int_{0}^{\infty} \mathrm{H}(\zeta, \tau) \mathrm{e}^{-s \tau} \mathrm{d} \tau
$$

Taking the Laplace transforms of Eqs. (21-24), we get

$$
\begin{aligned}
& \bar{H}^{\prime \prime}-2 \mathrm{~s} \overline{\mathrm{H}}=2 \mathrm{e}^{-(1+\mathrm{i}) \zeta}, \\
& \overline{\mathrm{H}}(0, \mathrm{~s})=0(\tau>0), \\
& \overline{\mathrm{H}}(\infty, \mathrm{s})=0(\tau \geq 0),
\end{aligned}
$$

And we obtain the transformed solution as

$$
\overline{\mathrm{H}}=\frac{1}{\mathrm{~s}-\mathrm{i}} \mathrm{e}^{-\sqrt{2 \mathrm{~s} \zeta}}-\frac{1}{\mathrm{~s}-\mathrm{i}} \mathrm{e}^{-(1+\mathrm{i}) \zeta}
$$

In order to take the Laplace inversion of $\overline{\mathrm{H}}$, equation(29) becomes:

We obtain

$$
\overline{\mathrm{H}}=\frac{1}{2 \sqrt{\mathrm{s}}}\left(\frac{1}{\sqrt{\mathrm{s}}+\sqrt{\mathrm{i}}}+\frac{1}{\sqrt{\mathrm{s}}-\sqrt{\mathrm{i}}}\right) \mathrm{e}^{-\sqrt{2 \mathrm{~s} \zeta}}-\frac{1}{\mathrm{~s}-\mathrm{i}} \mathrm{e}^{-(1+\mathrm{i}) \zeta}
$$

$$
\mathrm{H}=\frac{1}{2} \mathrm{e}^{\mathrm{i} \tau}\left[\mathrm{e}^{\sqrt{2 i} \zeta} \operatorname{erfc}\left(\sqrt{\mathrm{i} \tau}+\frac{\zeta}{\sqrt{2 \tau}}\right)+\mathrm{e}^{-\sqrt{2 \mathrm{i} \zeta}} \operatorname{erfc}\left(-\sqrt{\mathrm{i} \tau}+\frac{\zeta}{\sqrt{2 \tau}}\right)\right]-\mathrm{e}^{\mathrm{i} \tau} \mathrm{e}^{-(1+\mathrm{i}) \zeta}
$$

Where erfc(.) denotes the complementary error function.

Using Eqs (20) and (31), one finds

$$
\frac{\mathrm{f}}{\Omega \mathrm{l}}+\mathrm{i} \frac{\mathrm{g}}{\Omega \mathrm{l}}=1-\mathrm{e}^{-(1+\mathrm{i}) \zeta}+\frac{1}{2}\left[\mathrm{e}^{\sqrt{2 \mathrm{i} \zeta}} \operatorname{erfc}\left(\sqrt{\mathrm{i} \tau}+\frac{\zeta}{\sqrt{2 \tau}}\right)+\mathrm{e}^{-\sqrt{2 \mathrm{i} \zeta}} \operatorname{erfc}\left(-\sqrt{\mathrm{i} \tau}+\frac{\zeta}{\sqrt{2 \tau}}\right)\right]
$$

The dimensionless shear stress components in the fluid are obtained by

$$
\frac{\mathrm{T}_{\mathrm{xz}}+\mathrm{iT}_{\mathrm{yz}}}{\mathrm{T}_{\mathrm{r}}}=\frac{\partial}{\partial \zeta}\left(\frac{\mathrm{f}}{\Omega \mathrm{l}}+\mathrm{i} \frac{\mathrm{g}}{\Omega \mathrm{l}}\right)
$$

Where $\mathrm{T}_{\mathrm{r}}=\sqrt{\mu \rho \Omega^{3} / 2} \mathrm{l}$, and $\mu$ is the dynamic viscosity of the fluid. Using equations (32-33), we obtain

$$
\begin{aligned}
& \frac{\mathrm{T}_{\mathrm{xz}}+\mathrm{iT}_{\mathrm{yz}}}{\mathrm{T}_{\mathrm{r}}}=(1+\mathrm{i})\left[\mathrm{e}^{-(1+\mathrm{i}) \zeta}+\frac{1}{2} \mathrm{e}^{\sqrt{2 i} \zeta} \operatorname{erfc}\left(\sqrt{\mathrm{i} \tau}+\frac{\zeta}{\sqrt{2 \tau}}\right)+\frac{1}{2} \mathrm{e}^{-\sqrt{2 i} \zeta} \operatorname{erfc}\left(-\sqrt{\mathrm{i} \tau}+\frac{\zeta}{\sqrt{2 \tau}}\right)\right] \\
& -\frac{2}{\sqrt{2}} \frac{1}{\sqrt{\pi \tau}}\left[\mathrm{e}^{\sqrt{2 \mathrm{i} \zeta}-\left(\sqrt{\mathrm{i} \tau}+\frac{\zeta}{\sqrt{2 \tau}}\right)^{2}}+\mathrm{e}^{\left.-\sqrt{2 \mathrm{i} \zeta}-\left(-\sqrt{\mathrm{i} \tau}+\frac{\zeta}{\sqrt{2 \tau}}\right)^{2}\right]}\right.
\end{aligned}
$$

Finally, we have the dimensionless shear stress components on the disk in the following form:

$$
\left(\overline{\mathrm{T}}_{\mathrm{xz}}\right)_{\zeta=0}+\mathrm{i}\left(\overline{\mathrm{T}}_{\mathrm{yz}}\right)_{\zeta=0}=(1+\mathrm{i})\left\{1+\frac{1}{2}[\operatorname{erfc}(\sqrt{\mathrm{i} \tau})-\operatorname{erfc}(-\sqrt{\mathrm{i} \tau})]\right\}-\sqrt{\frac{2}{\pi \tau}} \mathrm{e}^{-\mathrm{i} \tau}
$$

Where $\overline{\mathrm{T}}_{\mathrm{xz}}=\frac{\mathrm{T}_{\mathrm{xz}}}{\mathrm{T}_{\mathrm{r}}}$ and $\overline{\mathrm{T}}_{\mathrm{yz}}=\frac{\mathrm{T}_{\mathrm{yz}}}{\mathrm{T}_{\mathrm{r}}}$

\section{Solution at small times}

Although the solution given above is exact, we shall search for another solution that is valid for small values of time. Thus, our purpose is to compare the results obtained in two different forms. We shall write Eq (29) as follows:

$$
\overline{\mathrm{H}}=\frac{1}{\mathrm{~s}} \frac{1}{(1-\mathrm{i} / \mathrm{s})}\left[\mathrm{e}^{-\varphi \zeta}-\mathrm{e}^{-(1+\mathrm{i}) \zeta}\right]
$$

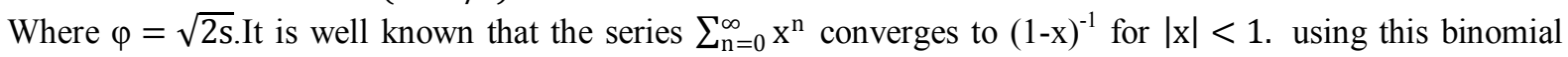
series, it is possible to obtain the solution for small values of the time corresponding to large s. (i.e.)

Or

$$
\overline{\mathrm{H}}=\frac{1}{\mathrm{~s}} \sum_{\mathrm{n}=0}^{\infty}\left(\frac{1}{\mathrm{~s}}\right)^{\mathrm{n}}\left[\mathrm{e}^{-\varphi \zeta}-\mathrm{e}^{-(1+\mathrm{i}) \zeta}\right]
$$

$$
\bar{H}=\sum_{n=0}^{\infty}(i)^{n} \frac{e^{-\varphi \zeta}}{s^{n+1}}-\sum_{n=0}^{\infty}(i)^{n} \frac{e^{-(1+i) \zeta}}{s^{n+1}}
$$

Now the Laplace inversion of $\overline{\mathrm{H}}$ becomes

$$
\mathrm{H}=\sum_{\mathrm{n}=0}^{\infty}(\mathrm{i})^{\mathrm{n}}(4 \tau)^{\mathrm{n}} \mathrm{i}^{2 \mathrm{n}} \operatorname{erfc}\left(\frac{\zeta}{\sqrt{2 \tau}}\right)-\mathrm{e}^{-(1+\mathrm{i}) \zeta} \mathrm{e}^{\mathrm{i} \tau}
$$

Where $i^{n} \operatorname{erfc}($.) denotes the repeated integrals of the complementary error function and is given by

$$
\mathrm{i}^{-1} \operatorname{erfc} \mathrm{x}=\frac{2}{\sqrt{\pi}} \mathrm{e}^{-\mathrm{x}^{2}}, \mathrm{i}^{0} \operatorname{erfc} \mathrm{x}=\operatorname{erfc} \mathrm{x},
$$




$$
\mathrm{i}^{\mathrm{n}} \operatorname{erfc} \mathrm{x}=\int_{\mathrm{x}}^{\infty} \mathrm{i}^{\mathrm{n}-1} \operatorname{erfc} \emptyset \mathrm{d} \emptyset(\mathrm{n}=0,1,2, \ldots)
$$

From equations (20) and (39), we have

$$
\frac{\mathrm{f}}{\Omega \mathrm{l}}+\mathrm{i} \frac{\mathrm{g}}{\Omega \mathrm{l}}=1-\mathrm{e}^{-(1+\mathrm{i}) \varsigma}+\mathrm{e}^{-\mathrm{i} \tau} \sum_{\mathrm{n}=0}^{\infty}(\mathrm{i})^{\mathrm{n}}(4 \tau)^{\mathrm{n}} \mathrm{i}^{2 \mathrm{n}} \operatorname{erfc}\left(\frac{\zeta}{\sqrt{2 \tau}}\right)
$$

Or

$$
\begin{gathered}
\frac{\mathrm{f}}{\Omega \mathrm{l}}=\left[1-\mathrm{e}^{\zeta} \cos \zeta\right]+(\cos \tau)[\mathrm{A}(\zeta, \tau)]+(\sin \tau)[\mathrm{B}(\zeta, \tau)], \\
\frac{\mathrm{g}}{\Omega \mathrm{l}}=\left[\mathrm{e}^{-\zeta} \sin \zeta\right]+(\cos \tau)[\mathrm{B}(\zeta, \tau)]-(\sin \tau)[\mathrm{A}(\zeta, \tau)],
\end{gathered}
$$

Where

$$
\begin{aligned}
& {[\mathrm{A}(\zeta, \tau)]=\operatorname{erfc}\left(\frac{\zeta}{\sqrt{2 \tau}}\right)-(4 \tau)^{2} \mathrm{i}^{4} \operatorname{erfc}\left(\frac{\zeta}{\sqrt{2 \tau}}\right)+(4 \tau)^{4} \mathrm{i}^{8} \operatorname{erfc}\left(\frac{\zeta}{\sqrt{2 \tau}}\right)-\cdots,} \\
& {[\mathrm{B}(\zeta, \tau)]=(4 \tau) \mathrm{i}^{2} \operatorname{erfc}\left(\frac{\zeta}{\sqrt{2 \tau}}\right)-(4 \tau)^{3} \mathrm{i}^{6} \operatorname{erfc}\left(\frac{\zeta}{\sqrt{2 \tau}}\right)+(4 \tau)^{5} \mathrm{i}^{10} \operatorname{erfc}\left(\frac{\zeta}{\sqrt{2 \tau}}\right)-\cdots,}
\end{aligned}
$$

Bearing in mind the identity

One finds

$$
\frac{d}{d z} i^{n} \operatorname{erfc} y=-\left(\frac{d y}{d z}\right) i^{n-1} \operatorname{erfc} y,
$$

$$
\begin{aligned}
& \frac{\mathrm{T}_{\mathrm{xz}}}{\mathrm{T}_{\mathrm{r}}}=\mathrm{e}^{-\zeta}(\cos \zeta+\sin \zeta)-\frac{\cos \tau}{\sqrt{2 \tau}}[\mathrm{C}(\zeta, \tau)]-\frac{\sin \tau}{\sqrt{2 \tau}}[\mathrm{D}(\zeta, \tau)], \\
& \frac{\mathrm{T}_{\mathrm{yz}}}{\mathrm{T}_{\mathrm{r}}}=\mathrm{e}^{-\zeta}(\cos \zeta-\sin \zeta)-\frac{\cos \tau}{\sqrt{2 \tau}}[\mathrm{D}(\zeta, \tau)]+\frac{\sin \tau}{\sqrt{2 \tau}}[\mathrm{C}(\zeta, \tau)],
\end{aligned}
$$

where

$$
\begin{aligned}
& {[\mathrm{C}(\zeta, \tau)]=\mathrm{i}^{-1} \operatorname{erfc}\left(\frac{\zeta}{\sqrt{2 \tau}}\right)-(4 \tau)^{2} \mathrm{i}^{3} \operatorname{erfc}\left(\frac{\zeta}{\sqrt{2 \tau}}\right)+(4 \tau)^{4} \mathrm{i}^{7} \operatorname{erfc}\left(\frac{\zeta}{\sqrt{2 \tau}}\right)-\cdots,} \\
& {[\mathrm{D}(\zeta, \tau)]=(4 \tau) \mathrm{i} \operatorname{erfc}\left(\frac{\zeta}{\sqrt{2 \tau}}\right)-(4 \tau)^{3} \mathrm{i}^{5} \operatorname{erfc}\left(\frac{\zeta}{\sqrt{2 \tau}}\right)+(4 \tau)^{5} \mathrm{i}^{9} \operatorname{erfc}\left(\frac{\zeta}{\sqrt{2 \tau}}\right)-\cdots,}
\end{aligned}
$$

Using the identity

$$
\mathrm{i}^{\mathrm{n}} \operatorname{erfc} 0=\left[2^{\mathrm{n}} \Gamma\left(\frac{\mathrm{n}}{2}+1\right)\right]^{-1}(\mathrm{n}=-1,0,1,2, \ldots)
$$

the shear stress components on the disk are

$$
\begin{aligned}
\left(\mathrm{T}_{\mathrm{xz}}\right)_{\zeta=0}=1-\frac{\cos \tau}{\sqrt{2 \tau}}\left[\frac{1}{2^{-1} \Gamma(0.5)}-\right. & \left.\frac{(4 \tau)^{2}}{2^{3} \Gamma(2.5)}+\frac{(4 \tau)^{4}}{2^{7} \Gamma(4.5)}-\frac{(4 \tau)^{6}}{2^{11} \Gamma(6.5)}+\cdots\right] \\
\quad-\frac{\sin \tau}{\sqrt{2 \tau}}\left[\frac{(4 \tau)}{2 \Gamma(1.5)}-\frac{(4 \tau)^{2}}{2^{5} \Gamma(3.5)}+\frac{(4 \tau)^{4}}{2^{9} \Gamma(5.5)}-\frac{(4 \tau)^{6}}{2^{13} \Gamma(7.5)}+\cdots\right], & \\
\left(\mathrm{T}_{\mathrm{yz}}\right)_{\zeta=0}=1-\frac{\cos \tau}{\sqrt{2 \tau}}\left[\frac{(4 \tau)}{2 \Gamma(1.5)}-\right. & \left.\frac{(4 \tau)^{2}}{2^{5} \Gamma(3.5)}+\frac{(4 \tau)^{4}}{2^{9} \Gamma(5.5)}-\frac{(4 \tau)^{6}}{2^{13} \Gamma(7.5)}+\cdots\right] \\
& -\frac{\sin \tau}{\sqrt{2 \tau}}\left[\frac{1}{2^{-1} \Gamma(0.5)}-\frac{(4 \tau)^{2}}{2^{3} \Gamma(2.5)}+\frac{(4 \tau)^{4}}{2^{7} \Gamma(4.5)}-\frac{(4 \tau)^{6}}{2^{11} \Gamma(6.5)}+\cdots\right],
\end{aligned}
$$

where $\Gamma($.$) is the gamma function defined by$

$$
\Gamma(n)=\int_{0}^{\infty} x^{n-1} e^{-x} d x(n>0)
$$

For small values of time, the solutions given by equations (42-43) and (52-53) can be used instead of the exact solutions given by Eqs. (32) and (35), respectively. It is clear that equations(42-43) and (52-53) are very convenient for $\tau \leq 0.25$.

\section{Results and Discussion}

When a disk and a fluid at infinity rotate with the same angular velocity about non-coincident axes, the fluid layer in each $\mathrm{z}=$ constant plane rotates as a rigid body with their angular velocity. The coordinates of the rotation centers of these fluid layers in this paper are obtained by $\frac{\mathrm{x}}{\mathrm{l}}=-\mathrm{g} / \Omega \mathrm{l}$ and $\frac{\mathrm{y}}{\mathrm{l}}=-\mathrm{f} / \Omega \mathrm{l}$ for $0 \leq \zeta<$ $\infty$. Thus the velocity components in the planes parallel to the xy-plane are calculated according to the coordinates.

In our problem, the disk and the fluid at infinity are initially rotating with the same angular velocity about two parallel axes normal to the disk. Hence, the initial condition becomes the solution given by Corier(1972). Because of the rotation about the common axis for $t>0$, the fluid tends to a rigid body rotation and 
it rotates about the $\mathrm{z}^{\prime}$-axis in the steady flow, as expected (Figures 1-2). In the limit as $\tau \rightarrow \infty$, this result is readily acquired from Eq. (41) for every $\zeta$.

At the region near the disk, the projections of the rotation enter of the fluid layers on the xy-plane are in the second quadrant until the computed value $\tau=2.05158$. After this instant, the projections are in the first quadrant. The projections continue to be in both the first and second quadrants as time elapses, and they reach the point o $(0,1,0)$ in the steady flow.

Since the shear stress components $\mathrm{T}_{\mathrm{xz}}$ and $\mathrm{T}_{\mathrm{yz}}$ do not depend on $\mathrm{x}$ and $\mathrm{y},\left(\mathrm{T}_{\mathrm{xz}}\right)_{\zeta=0}$ and $\left(\mathrm{T}_{\mathrm{yz}}\right)_{\zeta=0}$ are related to the $\mathrm{x}$ and $\mathrm{y}$-components of the force per unit area exerted by the fluid on the disk ,respectively .At small times ,the components become negative and positive in the $\mathrm{x}$ - and $\mathrm{y}$-directions, respectively; however, the $\mathrm{x}$-component is larger than the $\mathrm{y}$-component. The components change their directions continuously and finally go to zero. As shown in Figure 4, these components become equal at some values of time (for example $\left.\left(\mathrm{T}_{\mathrm{xz}}\right)_{\zeta=0}=\left(\mathrm{T}_{\mathrm{yz}}\right)_{\zeta=0}=0.11928\right)$ for $\tau=1.37947$ and $\left.\left(\mathrm{T}_{\mathrm{xz}}\right)_{\zeta=0}=\left(\mathrm{T}_{\mathrm{yz}}\right)_{\zeta=0}=-0.02951\right)$ for $\left.\tau=4.22331\right)$.

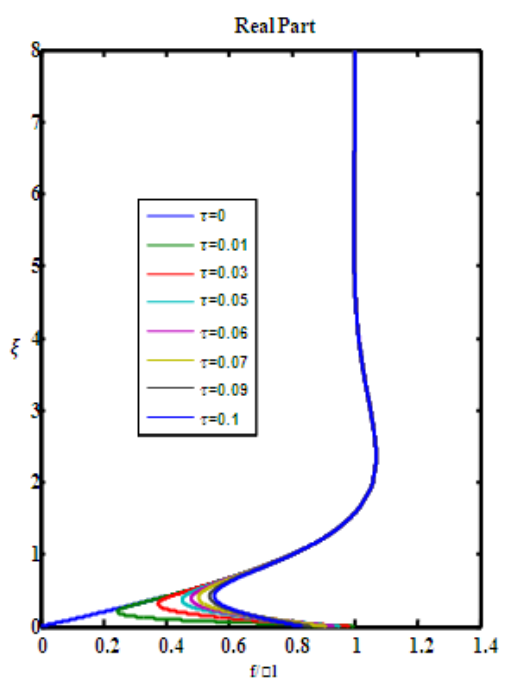

Figure 1 : variation of $f / \Omega l$ versus $\xi$ for various values of $\tau$

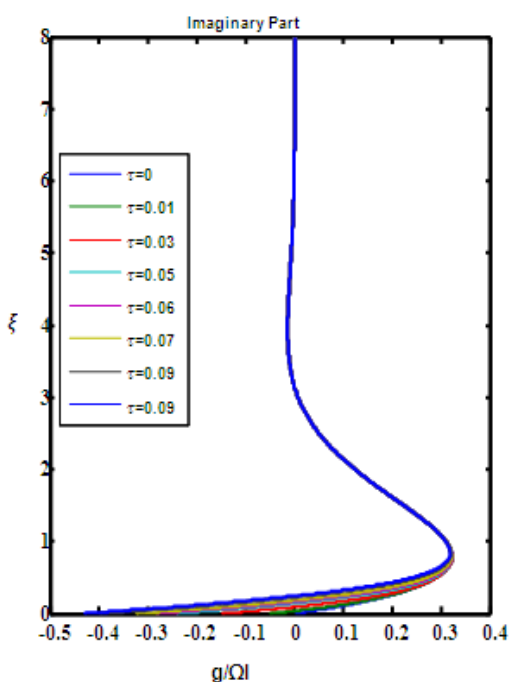

Figure 2: Variation of $g / \Omega$ l versus $\xi$ for various values of $\tau$

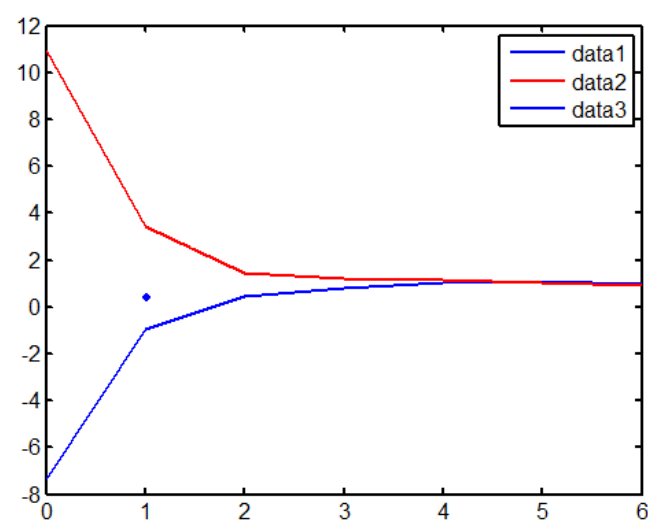

\section{Conclusion}

The unsteady viscous flow for produced by a sudden coincidence of the axes of a disk and the fluid at infinity rotating with the uniform angular velocity $\Omega$ has been studied. Exact solution of the governing equation has been obtained using Laplace transform technique are analysis to produced the result. It is found that the fluid tense to uniform motion in the curse of time $\tau>0$.

\section{Reference}

[1]. Abramowitz, M., and Stegun, I.A., Handbook of Mathematical Functions, Dover Publications, NewYork, 1965.

[2]. Berker, R., \An Exact Solution of the Navier-Stokes Equation: The Vortex with Curvilinear Axis", Int.J. Eng. Sci., 20, 217-230, 1982 . 
[3]. Coirier, J., \Rotations non Coaxiales d'un Disque etd'un Fluide_a L'in_ni", J. M_ecanique, 11, 317-340,1972.

[4]. Erdo gan, M.E., \Flow due to Eccentric Rotating a Porous Disk and a Fluid at In nity", J. Appl. Mech. - T. ASME,43E, 203-204, 1976 .

[5]. Erdo_gan, M.E., Non-Newtonian Flow due to Noncoaxially Rotations of a Disk and a Fluid at In_nity", Z. Angew.Math. Mech., $56,141-146,1976 b$.

[6]. Erdo_gan, M.E., IFlow due to Noncoaxially Rotations of a Porous Disk and a Fluid at In_nity", Rev. Roum. Sci.Techn.- M_ec. Appl., 22, 171-178, 1977.

[7]. Erdo_gan, M.E., IUnsteady Flow of a Viscous Fluid due to Non-Coaxial Rotations of a Disk and a Fluid at In_nity", Int. J. NonLinear Mech:, 32, 285-290, 1997.

[8]. Erdo_gan, M.E., \Flow Induced by Non-Coaxial Rotation of a Disk Executing Non- Torsional Oscillations and a Fluid Rotating at In_nity", Int. J. Eng.Sci., 38, 175-196, 2000.

[9]. K. R. Rajagopal, "On the flow of a simple fluid in an orthogonal rheometer," Archive for Rational Mechanics and Analysis, vol. 79, no. 1 , pp. 39-47, 1982.

[10]. T. Hayat, R. Ellahi, S. Asghar, and A. M. Siddiqui, "Flow induced by non-coaxial rotation of a porous disk executing non-torsional oscillations and a second grade fluid rotating at infinity," Applied Mathematical Modelling, vol. 28, no. 6, pp. 591-605, 2004. 